\title{
Microwave Nondestructive Testing Method
}

\author{
V. I. Gorbunov \& V. A. Sutorikhin \\ Remote Indicators of Active Defects (RIAD) Ltd. Co. \\ E-mail: wind@mail.tomsk.ru
}

Received: November 9, 2011

Accepted: December 5, 2011 Published: February 1, 2012

doi:10.5539/apr.v4n1p206

URL: http://dx.doi.org/10.5539/apr.v4n1p206

\begin{abstract}
The processes of ultrasonic elastic wave interaction with conduction electrons in metals were investigated by a new technique- microwave (MW) sensor. The authors obtained practical application results in the nondestructive testing (NDT) of metal objects in the static and motion mode.
\end{abstract}

Keywords: Microwave (MW) sensor, Nondestructive testing (NDT), Conduction electrons, Ultrasonic elastic wave

\section{Introduction}

The historical development of nondestructive testing (NDT) methods as an important science involves not only the formulation of new concepts, but also the development of modern techniques. Over the past decades there have been numerous research papers and manuals devoted to the problem of those processes emerging in metals under the impact of external factors. One such example is the investigation of metals affected by variable magnetic fields (Pustovoid V. I., 1969; Lifshitz I. M., et al., 1977; Schoenberg D., 1986; Tucker J., \& Rampton V., 1975; Tavger B.A., \& Demikhovsky V. Ya., 1968; Ando T., Fowler A, \& Stern F., 1985), where the basic problem -task was to determine the metal properties in accordance to its skin layer. Based on these investigations the conduction electron energy ("Fermi surface" energy) of alkali metals, Bi and others was calculated.

Practical application of these investigations included the determination of high-frequency measurements. Such methods as eddy current (RD-13-03-2006, p10) and thermography inspection (RD-13-03-2006, p11) involved those experimental attempts to apply surface conduction properties in analyzing the metal mechanical properties. These methods revealed the fact that local metal inhomogeneities are associated with surface cracks, casting flaws and mill defects.

Acoustic emission (AE) testing based on the application of the so-called "tin cry" phenomenon (http://www.zetms.ru/catalog/analyzers/control; GOST 27655-89), pinpointed such parameters as durability, fatigue life of metals and the dangerous zones on the surface and inside the metal itself. This method found a wide application in the research of metal and alloy reliability, investigation of low-durability zones producing acoustic waves by the release of localized stress energy (http://www.zetms.ru/catalog/analyzers/control; GOST 27655-89).It was presupposed that these acoustic waves are the first factors that determine the dynamic defects of the experimental object. Until recently, the functional relationship of the surface conduction to the ultimate internal stress was unknown, excluding the discovery- Electromagnetic Radiation during Crack Propagation in Metals by prof. A., Misra (Misra A., \& Kumar A., 2004). Prof. A., Misra research, related to a contactless registration of radio emission (propagation) during crack formation in metals, had no practical application. However, this research could have been relatively close to the acoustic emission method, in the case, if it was possible to register initial metal failure by the contactless method. Nowadays the method where the investigated object is in performance under mechanical stress could be a variant of the nondestructive testing.

The majority of modern metal sensing methods is in a steady-state regime functioning in a fixed positioning of the object itself, and in its turn, includes the following steps- shutdown and temporary production process shutoff. Taking into account the maintenance expenses the price of the aging equipment increases, respectively. Furthermore, as operation losses increase this involves the implementation of dynamic testing methods to exclude shutdown. Although suggested nondestructive testing method is similar to AE method in reliability and performance potential, it implements the following requirements- operates under specific working load, excludes shutdown, performs in operation regimes or, ideally, through continuous control of equipment loading rate. The proposed NDT method is similar to the AE method, but different in its dynamic research possibilities; and 
similar to prof. A. Misra method, but based on a new physical phenomenon- the interaction of electron gas and ultrasonic waves in metals. We developed a new technique for NDT and began its limited production.

\section{Research Background}

To apply the remote measurement method for AE parameters, emission of ultrasonic waves on the metal surface was determined by microwave sensor (Doppler radar). According to researchers (http://www.zetms.ru/catalog/analyzers/control; GOST 27655-89; Misra A., \& Kumar A., 2004; http://fizfiz1.narod.ru/101-139/102.htm; http://www.pacndt.com/index; Muraviov T. P., Zuev L. B., 2008; Muraviov V. V., et al., 2003; Vasilyev B. V., \& Lyuboshitz V . L., 1994) the obvious processes of elastic wave penetration into metals should indicate the fact that within and at the surface of metal zones there are different densities and different electrical conductions. However, after numerous experiments, the authors of this paper could not register the alternating component of surface metal conduction affected by ultrasonic waves. The spectral structure of microwave frequency reflected from the metal surface, does not include components with ultrasonic frequency. At the same time, the metal surface remained as an n-linear function of incident wave transmission, where the ultrasonic reflection output is less than $5 \mathrm{~W} / \mathrm{cm}^{2}$, while microwave frequency field output is not more than $5 \mathrm{~mW} / \mathrm{cm}^{2}$. Even today this problem has not been solved, notwithstanding the numerous conflicting explanations (http://www.pacndt.com/index; Muraviov T. P., Zuev L. B., 2008; Muraviov V. V., et al., 2003). It is the variety of explanations that makes it impossible to choose the accurate concept in this case. Nevertheless, the newly revealed phenomenon could be the only answer to this question.

It was in 1995 that the authors revealed those conditions under which the ultrasonic-induced metal surface modulated the reflected microwave frequency to phases. The following experiment was conducted in tension testing device: microwave (MW) sensor (designed in accordance to Doppler radar) was focused on the investigated surface (120-180mm), simultaneously switching on the ultrasonic transmitting unit (frequency 47 $\mathrm{KHz}$ ) - a simulator of ultrasonic (flexural) waves. The testing tension sample was ST 45 brand- steel (3x $45 \times 450 \mathrm{~mm}$ ). Furthermore, spectrum analyzer system SK 4-59, plugged into the microwave (MW) sensor outlet in the "standby" mode, registered spectral ultrasonic frequency component of $20 \mathrm{decibel}$ above noise level. The noise level of spectrum analyzer system SK4-59 is determined according to the performance instructions and includes -138 decibel relative to milliwatt for the investigated band of $1 \mathrm{KHz}$ and noise coefficient of about 6 decibel at $20^{\circ} \mathrm{C}$. Moreover, even before the experiment, the spectral ultrasonic components, exceeding the noise level, could not be registered. These amazing results led to further testing experiments with other samples - steel, aluminum, brass and copper ones. The following tests, conducted in accordance to the above-mentioned procedure description, confirmed the fact that the unknown observed phenomenon was not accidental.

It was established that this phenomenon is associated with dynamic defects in the investigated samples. Dynamic defects according to GOST requirements in AE (GOST 27655-89) are defined are those defects exhibiting activity (generating ultrasonic impulses) under stress. The "counting rate" for such defects is proportional to the imposed load (GOST 27655-89). It was experimentally established that the location of active defects (beginning of micro-crack formation) is not interconnected with the possible registration location of alternating surface conduction. The entire surface conduction of the investigated metal is variable. The microwave (MW) sensor (updated Doppler radar) detected spectral ultrasonic wave component at any point on the metal surface, reflecting the impinging microwave frequency energy. It was apparent that AE signals from active defects could be remotely registered, i.e. at such a distance where reflected microwave frequencies could be received. At the same time acoustic signals determined by AE method are secondary ones. Electric field signals (alternating conduction), travelling at the speed of light within the metal, significantly surpass those of acoustic signals, travelling at the speed of sound.

Theoretical evidence describing this newly observed phenomenon could be found in the research works of the scientists from Kurchatov Institute (Vasilyev B. V., \& Lyuboshitz V. L., 1994). Based on the well-known Virial theorem, they proved that within a metal, in a degenerated electron gas medium, subjected to physical stress (compression or tension), electrical-charged zones could form. The occurrence of inner electrical field is enhanced by the alteration of the surface charge structures, and the generation of surface electrical field opposite in sign. It is well-known that the propagation speed of an electrical field in an electron gas medium occurs at the speed of light (http://fizfiz1.narod.ru/101-139/102.htm; Bredow M. M., Rumyantsev V. V., \& Toptigin I. N., 1985; Vasilyev B. V., \& Lyuboshitz V. L., 1994). Therefore, the compensating surface field, alternating the surface conduction, occurs practically simultaneously all over this surface (Bredow M. M., Rumyantsev V. V., \& Toptigin I. N., 1985; Vasilyev B. V., \& Lyuboshitz V. L., 1994). Thus, the theoretical conclusions in the above-mentioned article (Vasilyev B. V., \& Lyuboshitz V. L., 1994) fundamentally verify our experimental results. 
Presupposing that the strain (compression or tension) is interconnected with the response of elastic ultrasonic waves, and then it could be stated that alternating surface conduction component (to the tenths of one percent) is linear-related to the amplitude of the wave itself (Vasilyev B. V., \& Lyuboshitz V. L., 1994). The formation zone of alternating conduction involves the entire metal surface which was also experimentally proved (Gorbunov V. I., \& Sutorikhin V. A., 1999).Our experiments not only gave adequate support for previous theoretical conclusions, but also forwarded such important results as the interconnection of spectral component amplitude to active defect magnitude, their size and positioning, defect parameters, resulting from extreme stress factors, which in its turn, proved the practical application of this phenomenon. The most significant conclusion based on the theoretical works of Moscow scientists (Vasilyev B. V., \& Lyuboshitz V. L., 1994) is the possible fact to determine remotely dangerous-or-non-dangerous inner metal stress, measuring the surface conduction (density of surface charges).

Several patents were filed in Russia (Gorbunov V. I., Sutorikhin V. A., \& Ustinov B. F., 2008a; 2008b; Brichkov S. A., Sutorikhin V. A., 2008), as well as, publications (Gorbunov V. I., \& Sutorikhin V. A., 1999; 23. Gorbunov V. I., \& Sutorikhin V. A., 2010). "Remote Indicators of Active Defects" Ltd. Co. was established by the authors. During the past two years (2010-2011 federal program "Start-10") research and testing were conducted, including remote indicators of active defects (RIAD) on steel samples and railway wheels in static mode; as well as, dynamic testing in moving mounted wheels. The first year program was completed successfully.

\section{Experiment Results}

The authors of this paper designed a laboratory unit of remote active defect indication in metals- microwave (MW) senor unit with frequencies between 10-37 GHz, registering alternating surface conduction at a distance of $100-180 \mathrm{~mm}$. The unit includes an ultrasonic generator with contact piezoconverters with frequencies between 44-400 KHz, RODE\&SH spectral analyzer or domestic SK4-59, AKTAKOM (ASK-3106) analog-to-digital converter, $h p$ computer, special simulator of active defects to regulate and monitor microwave frequency senor. This installation was designed as laboratory equipment. To increase the response a sensor with frequency of $37 \mathrm{GHz}$ was used, whereas, a sensor with frequency of $10 \mathrm{GHz}$ has less response and is less expensive. The first-variant sensors were designed on the basis of two devices- transmitter (Gunn diode) and receiver (semiconductor Schottky diode). The more complex variants were designed on the basis of heterodyning, phase comparison of emitted and received signal in the frequency interval $(100-400 \mathrm{MHz})$. This complex variant makes it possible to avoid dead spots of microwave (MW) sensor due to stationary waves (SW). Average microwave (MW) senor response is $0.2-0.3 \%$ of the surface conduction, when target signal is 3decibel higher than noise for a band of $300-600 \mathrm{~Hz}$. Figures $1-4$ show the testing results where the simple sensor with frequency of $37 \mathrm{GHz}$ was used.

The spectrograms in Figure 1 and 2 show the elastic tension of an electric steel sample $(200 \times 35 \times 0.3 \mathrm{~mm})$. The spectrogram in Figure 1 shows a short impulse (not more than 1 second), registered by the spectral analyzer under elastic tension of the sample. The second spectrogram shows the signal registered during the slow tension relief of the same sample. The mechanical load level in the first and second case is relatively close. The experiment showed that this new control method could be used to determine the initial formation of active defects (cracks), even under elastic tension.

The next experiment stage was conducted on the flexural testing machine for ST- steel samples $(200 \times 25 \times 4 \mathrm{~mm})$ to determine the possible indications of fatigue cracking after numerous bending cycles (bending angle $\pm 30-35^{0}$ ). The indicator is SK4-59 with an AKTAKOM ASK 3106 adapter and $h p$ computer (Figures 3, 4).

The investigation results were similar to those observed in AE (GOST 27655-89; http://www.pacndt.com/index); however, in the following case, cracking (1-2mm) is accompanied by non-continuous prolonged signal (Fig.4) -several hours. It is known that $\mathrm{AE}$ signals from fatigue cracking cannot be registered without additional mechanical load (GOST 27655-89; http://www.pacndt.com/index).The experiment showed that target signals, registered by the new method, exist for a prolonged interval rendering reliable defect detection (high significance level).

Most of the research was devoted to testing of active defects in railway wheels in the static mode. Obtained results extended the possible potential performance of the new method. Previously unknown phenomenon was detected by using bi-harmonic ultrasonic signals; engineering calculation formulae for detecting active defect size, its positioning on the investigated object, including the object geometry itself were obtained, as well. Displayed signals determined the defect positioning to its amplitude, non-continuous development time-interval, "blinking" parameters, which were detected on the massive railway wheel samples. Defect reactivation methods have been developed, which in its turn, has furthered the application of the above-mentioned new method. 
Characteristic features of emerging displayed signals, their time parameters are rather similar to those parameters of the AE method (http://www.zetms.ru/catalog/analyzers/control; GOST 27655-89; http://www.pacndt.com/index).Direct comparison of $\mathrm{AE}$ signals and microwave (MW) sensor signals is practically impossible to detect as the additional ultrasonic source is significantly higher than the energy of possible AE signals. Nevertheless, the AE theory (GOST 27655-89; http://www.pacndt.com/index) and the practical comparison results (stated in Research Background) indicate the fact that AE signals and active defect signals, registered by microwave (MW) sensor, emerged simultaneously. In this case, simultaneity is the origin time of the overstrained mechanical condition in a fraction of metal. Further, there are two moments- the microwave (MW) sensor detects the alternating conduction, because of additional ultrasonic exposure propagating at the speed of light within the metal; whereas, piezosensor receives a signal from the active zone at the speed of noise ( thousand times slower). Repeated experiments based on available test objects, including railway wheels, illustrated the new characteristic feature of the microwave (MW) sensor. Specific design features, microwave (MW) sensor schemes, impulse analog provide a means of designing and developing cost-effective and reliable indicators of active defects involved in the non-stop production process. The impulse regime of the ultrasonic generator is used to calculate the positioning of active defects in accordance to the delay time between the initial generation and the origin time of the skin layer conduction variation at the microwave (MW) sensor outlet.

\section{Conclusion}

Significant research and practical application of obtained research results are of great interest and importance for engineers and professionals. The pilot project planning relevant to R\&D requirements pinpoints the further implementation of this new nondestructive testing method into industrial production. The present indicators of active defects have several variations which are being updated to implement the testing technique for railway wheels (in motion mode) into limited production. The existing difficulties and investment problems are associated with the slight involvement of domestic consumers.

Completed research showed the possible range increase in this method package; whereas the existing operating range is limited due to the fact that the microwave field focuses only on one selected surface (Bredow M. M., Rumyantsev V. V., \& Toptigin I. N., 1985). It is possible to use this method package in investigating railway bridges (as the AE method is already applied (Muraviov V. V., et al., 2003)), moving objects- for example, turbine blades, pump shaft assemblies and propeller blades. Although we are not revealing the design details of our know-how, we would be ready for any partnership or future buyer of this method package.

\section{References}

Acoustic Emission Measurement Technologies from Physical Acoustics. [Online] Available: http://www.pacndt.com/index

Ando T., Fowler A, \& Stern F. (1985). Electronic properties of two-dimensional systems (translation). p. 132.

Bredow M. M., Rumyantsev V. V., \& Toptigin I. N. (1985). Classical Electrodynamics. /Under the editorship of Toptigin I. N. M.: Nauka, 400 p.

Brichkov S. A., \& Sutorikhin V. A. (2008). Useful model patent No.-85390. The appliance for precision positioning during the surface treatment of metal products. Priority of a useful model. December 01 .

Gorbunov V. I., \& Sutorikhin V. A. (1999). Possible control of elastic deformation limits by the microwave (MW) method. Defectoscopy, No. 7, pp.75-80.

Gorbunov V. I., \& Sutorikhin V. A. (2010). Possibility of non-destructive testing using microwaves. Electronic Journal, Technical Acoustics. http://www.ejta.org, No.16, pp.9-18.

Gorbunov V. I., Sutorikhin V. A., \& Ustinov B. F. (2008a). Method patent No. 2372615. The method of registration of acoustic emission signals in metals. Priority of Invention. July 22.

Gorbunov V. I., Sutorikhin V. A., \& Ustinov B. F. (2008b). Useful model patent No. 81677. The device for detection of active defects in metal products. Priority of Invention, August 04.

GOST 27655-89. Acoustic Emission. Terms, definitions and designations.Guidelines in eddy current control procedure of technological devices and equipment applied and exploited in dangerous production sites./ RD-13-03-2006/ Edit.by K.B. Pulikovski / Put into operation from 25.12.06., P.10

Guidelines in thermal control procedure of technological devices and equipment applied and exploited in dangerous production sites. / RD-13-04-2006/Moscow, 2006, P.11. 
Lifshitz I. M., et al. (1977). The phenomenon of oscillations of thermodynamic and kinetic properties of solid body films. Discoveries. Inventions. Industrial designs. Trademarks, No.32, p. 3.

Misra A., \& Kumar A. (2004). Some basic aspects of electormagnetic radiation during crack propagation in metals. Intern. J. Fracture, V. 127, No. 4. P. 387-401. http://dx.doi.org/10.1023/B:FRAC.0000037676.32062.cb

Muraviov T. P., \& Zuev L. B. (2008). Features of acoustic emission during Chernov-Luders line development in low-carbon steel specimens. Journal of Technical Physics, Volume 78, Issue 8, 135-139.

Muraviov V. V., Boyarkin E. V., Vlasov K. V., Beher A., \& Kozyatnik I. I. (2003). Application of the acoustic emission method for inspection of bridges. Destruction and Monitoring of Metal Properties. The collection of works of the international conference/Machine Science Institute, Ekaterinburg, May 26-30, p. 85.

Pustovoid V. I. (1969). Interaction of electron flows with elastic waves of the lattice. Uspekhi Fizicheskikh Nauk, Vol. 97, Issue 2, p. 257.

Schoenberg D. (1986). Magnetic oscillations in metals (translation).

Tavger B.A., \& Demikhovsky V. Ya. (1968). Quantum dimensional effects in the semiconductors and submetallic films. Uspekhi Fizicheskikh Nauk, vol. 96, p. 61.

Tucker J., \& Rampton V. (1975). Hypersound in Solids (translation).

Vasilyev B. V., \& Lyuboshitz V. L. (1994). The Virial theorem and some properties of the electron gas in metals (RAS Session of February 23, 1994). Uspekhi Fizicheskikh Nauk, 4(164), pp. 367-374.

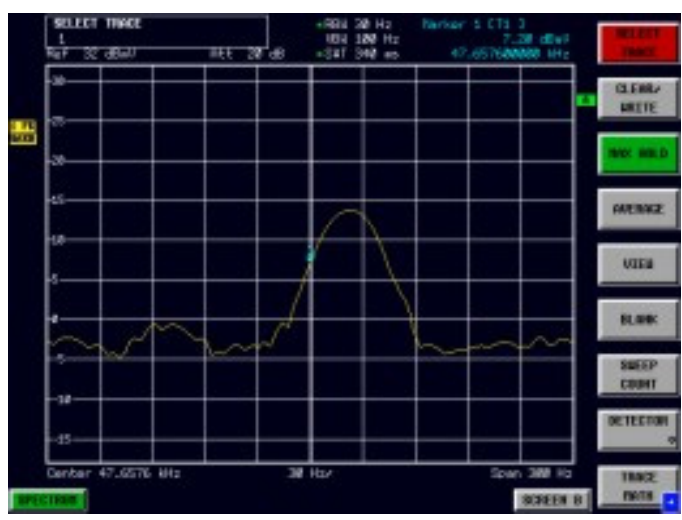

Figure 1. Spectrogram of electric steel sample microwave (MW) sensor with frequency of $37 \mathrm{GHz}$ (under tension)

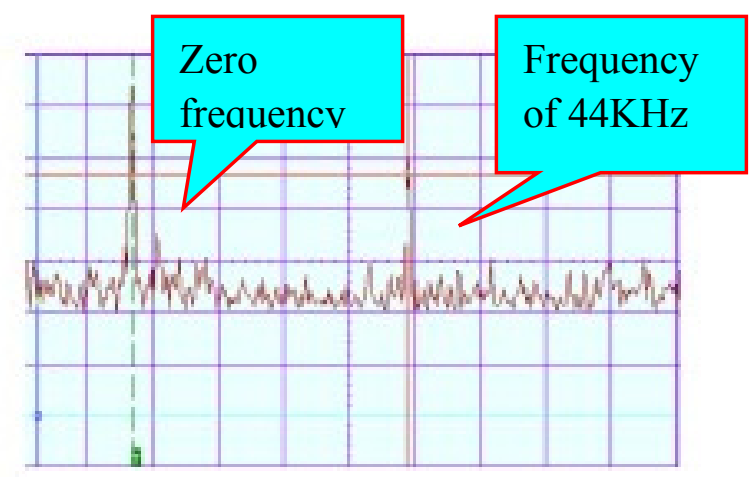

Figure 3. Spectrogram of sample before fatigue testing, microwave (MW) sensor with frequency of $37 \mathrm{GHz}$

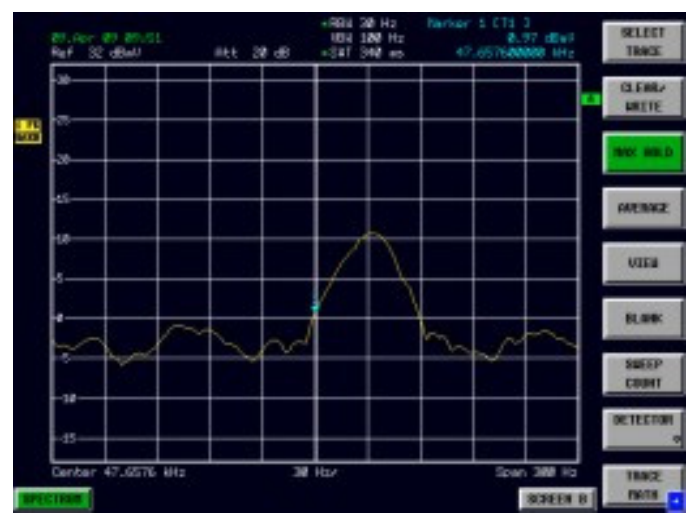

Figure 2. Spectrogram of electric steel sample microwave (MW) sensor with frequency of $37 \mathrm{GHz} 37 \mathrm{GHz}$ (tension relief)

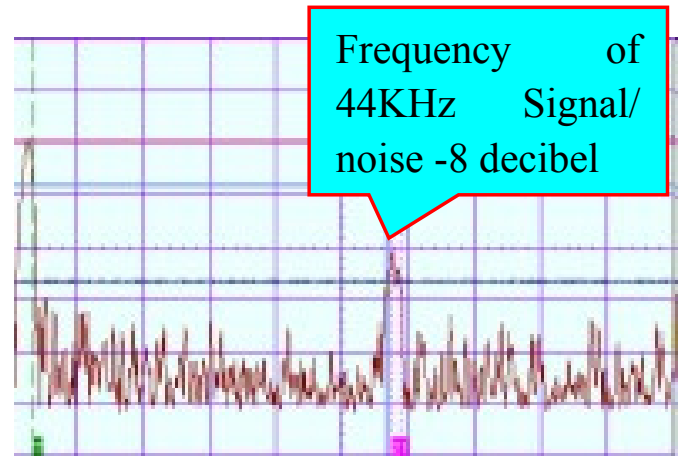

Figure 4. Spectrogram after 180 bends $\left(30^{0}-35^{0}\right)$

Cracking (1-2mm); microwave(MW) sensor 